\title{
Designing Homogeneous Bromine Redox Catalysis for Selective Aliphatic C-H Bond Functionalization
}

\author{
Peter Becker, ${ }^{[a,+]}$ Thomas Duhamel, ${ }^{[a, b,+]}$ Claudio Martínez, ${ }^{[a]}$ Kilian Muñiz ${ }^{* a, c]}$
}

\begin{abstract}
The potential of homogeneous oxidation catalysis employing bromine has remained largely unexplored. The combination of tetraalkylammonium bromide and 3-chloroperbenzoic acid offers a unique catalyst system for the convenient and selective oxidation of saturated $\mathrm{Csp}^{3}-\mathrm{H}$ bonds upon photochemical initiation with visible light. It enables remote intramolecular, position-selective $\mathrm{C}-\mathrm{H}$ amination as demonstrated for 20 different examples. For the first time, a $\mathrm{N}$-halogenated intermediate could be isolated as the active catalyst state in a catalytic Hofmann-Löffler reaction. In addition, a novel expeditious one-pot synthesis of $\mathrm{N}$ sulfonyloxaziridines from $\mathrm{N}$-sulfonamides was developed and exemplified for 15 transformations. These pioneering examples provide a change in paradigm for molecular catalysis with bromine.
\end{abstract}

The growing demand for environmentally benign oxidation conditions has placed catalysis with redox-active halide reagents into the focus of method development. While homogeneous catalysis based on the iodine $(I)$ platform is continuously maturing into a viable synthetic tool, ${ }^{[1]}$ related bromine catalysis has virtually remained in its infancy. We became interested in devising the as yet unprecedented bromine catalysis for aliphatic hydrocarbon functionalization reactions. In a seminal study, Sharpless had developed conditions for bromine-catalyzed aziridination of alkenes with chloramine-T under homogeneous conditions. ${ }^{[2]}$ Later, a bromine catalyzed intramolecular alkene diamination was reported. ${ }^{[3]}$ In amination chemistry, these reactions represent the presently small number of homogeneous bromine-catalyzed alkene oxidations, ${ }^{[4]}$ and the possibility of employing bromine as redox active homogeneous oxidation catalyst has been considered strongly limited. The usually low turn-over rates of homogeneous bromine catalysts have been suggested to be consequential of rapid catalyst deactivation (disproportionation and/or over-oxidation to a sink of unreactive $\mathrm{Br}^{\vee}$ species). ${ }^{[2 \mathrm{a}]}$ Here, we present the synthesis of pyrrolidines through a remote hydrocarbon activation of benzylic $\mathrm{C}-\mathrm{H}$ bonds mediated by a homogeneous bromide catalyst. The carefully elaborated conditions for such bromine catalysis represent a

[a] Dr. P. Becker, T. Duhamel, Prof. Dr. K. Muñiz Institute of Chemical Research of Catalonia (ICIQ), The Barcelona Institute of Science and Technology Av. Països Catalans 16, 43007 Tarragona (Spain) E-mail: kmuniz@iciq.es

[b] T. Duhamel

Facultad de Química, Universidad de Oviedo C/Julián Clavería, 33006 Oviedo (Spain)

[c] Prof. Dr. K. Muñiz

ICREA, Pg. Lluís Companys 23, 08010 Barcelona (Spain)

[+] These authors contributed equally.

Supporting information for this article is given via a link at the end of the document. uniform approach that is further applicable to a novel oxaziridine formation from sulfonamides. This work thus demonstrates the applicability of bromine catalysis in homogeneous oxidation chemistry without the loss of catalytic activity throughout the catalysis. Pyrrolidines and related saturated nitrogen-containing heterocycles are ubiquitous alkaloid scaffolds with outstanding biological and pharmaceutical activity, ${ }^{[5]}$ for which the direct amination of an aliphatic $\mathrm{C}-\mathrm{H}$ bond has been identified as a logical and economic strategy. ${ }^{[6,7]}$ Our recent development of related iodine catalyzed Hofmann-Löffler reactions (Figure 1$)^{[8]}$ has triggered our interest to develop related $\mathrm{Br}$-based protocols. In contrast to the existing paradigm, ${ }^{[2 a]}$ such an approach should be feasible taking into account historic parent transformations utilizing stoichiometric amounts of hypobromite as oxidizing reagent. ${ }^{[9,10]}$ Therefore, it would be particularly interesting as a proof of principle for bromine oxidation catalysis outside the few existing examples of alkene functionalization. ${ }^{[2-4,11]}$

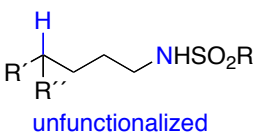
remote $\mathrm{C}-\mathrm{H}$ bond

lodine catalysis with an iodine(III) oxidant $\left(\mathrm{ArCO}_{2}\right)_{2} \mathrm{IPh}$ reagent as terminal oxidant

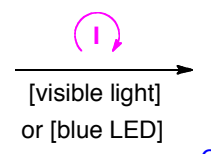

odine catalysis with a photoredoxbased terminal aerobic oxidation oxidant iodine catalys TPT (1 mol\%), air I-OH

Figure 1. Catalytic Hofmann-Löffler reactions based on molecular iodine as catalyst source. $\mathrm{Ar}=3-\mathrm{Cl}-\mathrm{C}_{6} \mathrm{H}_{4}$

We started our investigation applying conditions similar to the iodine-catalyzed Hofmann-Löffler reaction. ${ }^{[8 a]}$ When 1a was converted in the presence of elemental bromine $(10 \mathrm{~mol} \%)$ as the catalyst and bis(3-chlorobenzoyloxy)iodobenzene $\mathrm{Phl}\left(\mathrm{O}_{2} \mathrm{CAr}\right)_{2}$ ( 1.1 equiv.) as the oxidant, $22 \%$ of the desired pyrrolidine product $2 \mathrm{a}$ was obtained (Table 1 , entry 1 ). A change of the hypervalent iodine(III)-oxidant did not significantly improve the yield of $\mathbf{2 a}$ (entry 2 ). We then turned to a bromide salt, which can be administered as an ammonium derivative resulting in better solubility in organic solvents. The combination of tetrabutylammonium bromide with diacetoxy iodobenzene (PIDA) and bis(trifluoroacetoxy)iodobenzene (PIFA) increased the yield to 32 and $40 \%$, respectively (entries 3,4 ), while the best result was obtained with $\mathrm{Phl}\left(\mathrm{O}_{2} \mathrm{CAr}\right)_{2}$ (entry 5). Moreover, based on the particular effect of the 3-chlorobenzoate anion, more 
conventional $m$ CPBA (2 equiv.) was identified as an even better oxidant in combination with $20 \mathrm{~mol} \%$ of $\mathrm{Bu}_{4} \mathrm{NBr}$ (entry 6). The reaction reached full conversion and the cyclized compound $\mathbf{2 a}$ was isolated in $95 \%$ yield. The environmentally benign and economic conditions for $m C P B A$ as the oxidant deserve to be highlighted. The use of lower concentrations of either $\mathrm{Bu}_{4} \mathrm{NBr}$ or $m C P B A$ resulted in lower yields (entries 7-11). However, the reactions still produced reasonable amounts of product indicating significant robustness of the homogeneous bromine catalysis.

\begin{tabular}{|c|c|c|c|}
\hline \multicolumn{2}{|r|}{$1 \mathrm{a}$} & $\begin{array}{c}\begin{array}{c}\text { Br catalyst source } \\
\text { oxidant, }\end{array} \\
\text { solvent, } 12 \mathrm{~h}, 25^{\circ} \mathrm{C}\end{array}$ & \\
\hline Entry & Con & ditions & onversion $(\%)^{[a]}$ \\
\hline 1 & $\mathrm{Br}_{2}(10 \mathrm{~mol} \%), \mathrm{Phl}(\mathrm{C}$ & $\left.\mathrm{J}_{2} \mathrm{CAr}\right)_{2}$ (1.1 equiv.), DCE & 22 \\
\hline 2 & $\mathrm{Br}_{2}(10 \mathrm{~mol} \%), \mathrm{PIFA}$ & ( 1.1 equiv.), DCE & 30 \\
\hline 3 & $\mathrm{Bu}_{4} \mathrm{NBr}(20 \mathrm{~mol} \%)$ & PIDA (1.1 equiv.), $\mathrm{CH}_{3} \mathrm{CN}$ & 32 \\
\hline 4 & $\mathrm{Bu}_{4} \mathrm{NBr}(20 \mathrm{~mol} \%)$ & PIFA (1.1 equiv.), $\mathrm{CH}_{3} \mathrm{CN}$ & 40 \\
\hline 5 & $\mathrm{Bu}_{4} \mathrm{NBr}(20 \mathrm{~mol} \%)$ & $\mathrm{Phl}\left(\mathrm{O}_{2} \mathrm{CAr}\right)_{2}$ (1.1 equiv.), $\mathrm{CH}_{3} \mathrm{CN}$ & $N 46$ \\
\hline 6 & $\mathrm{Bu}_{4} \mathrm{NBr}(20 \mathrm{~mol} \%)$, & $m$ CPBA (2 equiv.), $\mathrm{CH}_{3} \mathrm{CN}$ & $100(95 \%)^{[b]}$ \\
\hline 7 & $\mathrm{Bu}_{4} \mathrm{NBr}(20 \mathrm{~mol} \%)$, & $m \mathrm{CPBA}$ (1.5 equiv.), $\mathrm{CH}_{3} \mathrm{CN}$ & 77 \\
\hline 8 & $\mathrm{Bu}_{4} \mathrm{NBr}(20 \mathrm{~mol} \%)$, & $m \mathrm{CPBA}$ (1.2 equiv.), $\mathrm{CH}_{3} \mathrm{CN}$ & 69 \\
\hline 9 & $\mathrm{Bu}_{4} \mathrm{NBr}(10 \mathrm{~mol} \%)$, & $m \mathrm{CPBA}$ (2 equiv.), $\mathrm{CH}_{3} \mathrm{CN}$ & 50 \\
\hline 10 & $\mathrm{Bu}_{4} \mathrm{NBr}(5 \mathrm{~mol} \%), m$ & CPBA (2 equiv.), $\mathrm{CH}_{3} \mathrm{CN}$ & 40 \\
\hline 11 & $\mathrm{Bu}_{4} \mathrm{NBr}(2.5 \mathrm{~mol} \%)$, & $m \mathrm{CPBA}$ (2 equiv.), $\mathrm{CH}_{3} \mathrm{CN}$ & s.m \\
\hline
\end{tabular}

Table 1. C-H Amination under Homogeneous Bromine Catalysis: Optimization. ${ }^{[a]}$ Estimated from integration in the ${ }^{1} \mathrm{H}$ NMR spectrum of the crude reaction mixture. ${ }^{[b]}$ Isolated yield of $2 a$ after purification. $\mathrm{Ar}=3-\mathrm{ClC}_{6} \mathrm{H}_{4}, \mathrm{PIDA}=$ $\mathrm{Phl}(\mathrm{OAC})_{2}, \mathrm{PIFA}=\mathrm{Phl}\left(\mathrm{O}_{2} \mathrm{CCF}_{3}\right)_{2}$.

The reaction conditions show generality for the formation of pyrrolidines (Scheme 1) and are applicable in the presence of electron withdrawing and electron donating groups on the arene affording the products $\mathbf{2 b}$-e with complete selectivity and in good yields $(68-95 \%)$. Different sulfonyl protecting groups at the nitrogen atom are well tolerated providing the respective nosylated (2f) and mesylated (2g) products in high yields (71$98 \%$ ). Also, a cyclopropylsulfonylamide is readily tolerated suggesting that potentially competing radical ring opening does not compete $(82 \%, 2 \mathrm{~h})$, while the 2-thiophenylsulfonylamide is likewise stable $(63 \%, 2 i)$. More congested 2- and 3-tosylgroups provide comparable yields to the standard 4-tosyl indicating that increased sterics do not interfere during the cyclisation (87 and $92 \%, \mathbf{2} \mathbf{j}$ and $\mathbf{2 k}$ ). Backbone modification is well tolerated $(94 \%$, 2I). Acyclic stereocontrol cannot be realized as demonstrated for compounds $\mathbf{2} \mathbf{m} / \mathbf{2} \mathrm{m}^{\prime}$, which produce a 1:1-mixture of diastereomers due to the radical reaction mechanism. However, cyclization within ring annulation provides single diastereomers 2 n $(79 \%)$ and $20(54 \%)$. The amination reaction in $\alpha$-position to a heteroatom also proceeds in good yields (54 and 98\%, 20 and $2 p)$. In addition, bromine catalysis tolerates benzylic tosylamides, which provide the isoindolines $\mathbf{2 q - 2 t}$ without imine formation, which is the dominating pathway in iodine catalysis. ${ }^{[8]}$ Heteroaromatic substituents do not undergo potentially competing bromination under these conditions (2r-t), and even the notoriously oxidation-labile furane core in $\mathbf{2 t}$ is tolerated entirely. These results demonstrate the first examples of a Hofmann-Löffler-type reaction under expedious homogeneous bromine catalysis. They significantly advance the harsh reaction requirements of the traditional process, which is stoichiometric in bromine promoter, requires a strong acidic reaction medium and a basic work-up. ${ }^{[9]}$

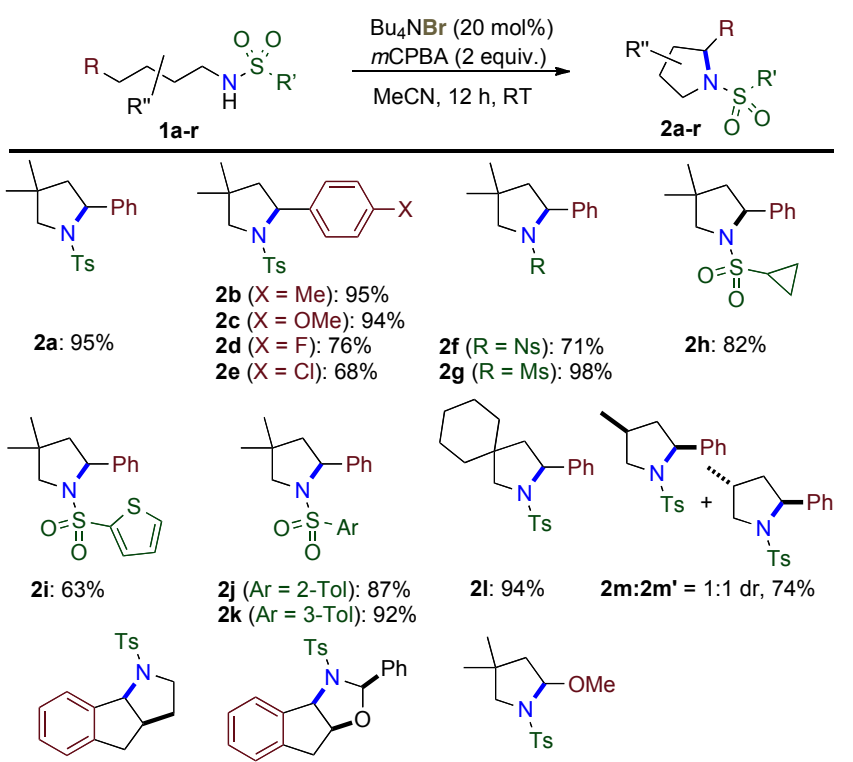

2n $1: 0 \mathrm{dr}, 79 \%$

$$
\text { 2o: } 1: 0 \mathrm{dr}, 54 \%
$$

2p: $98 \%$
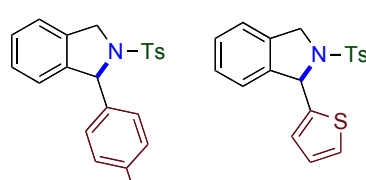

2r: $59 \%$

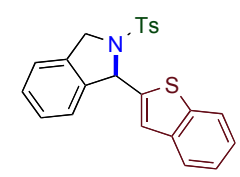

2s: $48 \%$

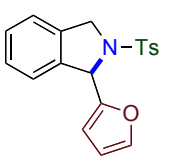

2t: $38(99) \%[a]$

Scheme 1. C-H Amination under Homogeneous Bromine Catalysis: Scope. ${ }^{[a]}$ Addition of $m C P B A$ in four portions. Yield in bracket based on isolated starting material.

Mechanistically, the reaction could proceed through various intermediary bromine(I) states, which derive from oxidation of the bromine source and could be either conventional hypobromite or 3-chlorobenzoyl hypobromite. Based on control experiments, we propose a catalysis that is based on 3chlorobenzoyl hypobromite 4 as the active catalyst (Scheme 2). This hypobromite 4 is directly formed from $\mathrm{Bu}_{4} \mathrm{NBr}$ and $m$ CPBA. ${ }^{[12]}$ Independent synthesis of the active catalyst $\mathbf{4}$ from the corresponding silver benzoate $3^{[13]}$ was successful, although 4 is of pronounced instability. Still, addition of 1 equivalent of standard substrate $1 \mathrm{a}$ to the solution of $\mathbf{4}$ provided a $24 \%$ formation of product $\mathbf{2 a}$ ( $48 \%$ normalized yield). In successive investigation, it was confirmed that hypobromite $\mathbf{4}$ indeed initiates catalysis, and a $65 \%$ overall formation of $\mathbf{2 a}$ was obtained in the presence of 2 equivalents of $m$ CPBA. The involvement of free hypobromite was excluded based on Raman spectroscopy. ${ }^{[14]}$ The involvement of a stabilized anionic species $\left[\operatorname{Br}\left(\mathrm{O}_{2} \mathrm{CAr}\right)_{2}\right]$ appears less likely. Such species had been previously isolated and documented to constitute electrophilic bromine sources in stoichiometric alkene oxidation reactions. ${ }^{[15]}$ We have generated such compounds by interaction between $\mathrm{Bu}_{4} \mathrm{NBr}$ and $\mathrm{PIFA}$ or $\mathrm{Phl}(m C B A)_{2}$, respectively, as they are 
presumably produced under the conditions from Table 1 , entries 4 and 5 . However, isolated anionic bromine(I) compounds do not show activity in the cyclization of $\mathbf{1 a}$, that would be comparable to the active catalyst $4 .^{[14]}$ This is an important mechanistic difference in behavior when compared to the corresponding iodine-based catalysts. ${ }^{22}$ In contrast to previous mechanistic investigations of iodine catalyzed Hofmann-Löffler reactions, ${ }^{[8]}$ it was possible to obtain the $\mathrm{N}$-brominated intermediate $\mathbf{5 a}$ from a reaction between $1 \mathrm{a}$, an ammonium bromide and $m \mathrm{CPBA}$. This synthesis resembles the formation of $\mathbf{5 a}$ under the real catalytic conditions. N-Brominated $\mathbf{5 a}$ could be unambiguously identified by NMR spectroscopy and by HRMS measurements displaying correct isotope pattern. It displays a remarkably high reactivity as evidenced from its estimated half-life time of $30 \mathrm{~min}$. The isolation of $\mathbf{5 a}$ constitutes the first detection and structural characterization of an $\mathrm{N}$-halogenated intermediate in halogencatalyzed Hofmann-Löffler reactions. In comparison to free 1a, the UV spectrum of $\mathrm{N}$-brominated $\mathbf{5 a}$ exemplifies a bathochromic shift to around $300 \mathrm{~nm}$, which is in agreement with a photochemical activation under normal environmental daylight. ${ }^{[14]}$ It also explains that reactions with LED light sources experience a strong decrease in yield. ${ }^{[14]}$ The required energy for $\mathrm{N}-\mathrm{Br}$ bond cleavage is significantly different from related iodinated species. ${ }^{[8 b]}$ This observation demonstrates that the development of light-initiated bromine oxidation catalysis cannot be predicted readily from the corresponding iodine catalysis.

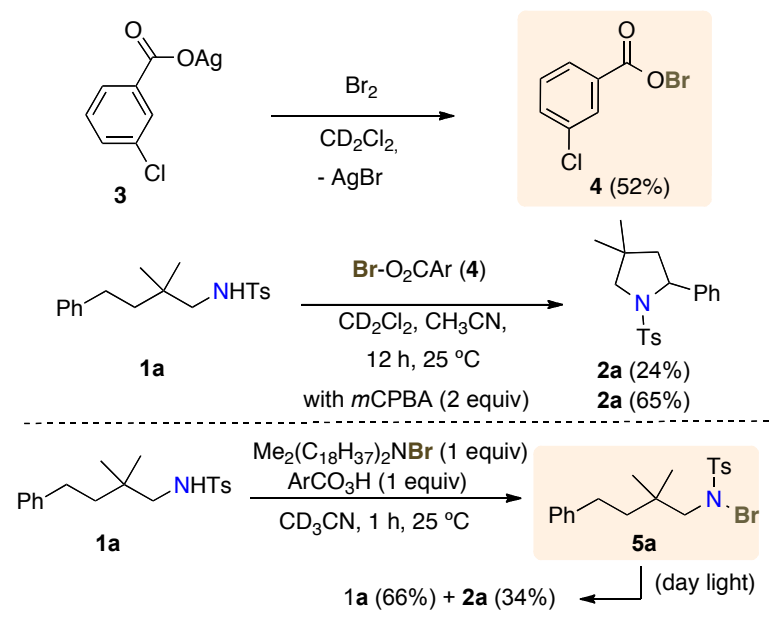

Scheme 2. Potential catalysts, control experiments and isolation of $\mathrm{N}$ brominated intermediate.

Under the catalytic conditions, the reaction between $\mathbf{4}$ and the substrate $\mathbf{1}$ provides the $\mathrm{N}$-brominated tosylamide $\mathbf{5}$, which undergoes light-induced homolysis to the $\mathrm{N}$ - centered radical intermediate A (Figure 2). The selectivity of the $\mathrm{C}-\mathrm{H}$ functionalization event arises from the involvement of a kinetically preferred $1,5-\mathrm{H}$ abstraction at this stage. A kinetic isotope effect of 3 was determined for this pathway as the ratedetermining step. The intermediary alkyl bromide $\mathbf{B}$ forms through either a radical chain mechanism involving 5, 3chlorobenzoyl hypobromite or from radical recombination. Given the occurrence of free $\mathbf{1 a}$ in the stoichiometric experiment with 5a from Figure 4, the former appears more reasonable. Subsequent nucleophilic cyclization requires the activated benzylic position or an $\alpha$-alkoxylation to provide a sufficiently fast reaction. This is the first case of direct pyrrolidine formation from $\mathrm{Br}$-based Hofmann-Löffler reactions as previous methods always terminate at the $\mathrm{C}-\mathrm{Br}$ stage requiring subsequent base administration for $\mathrm{C}-\mathrm{N}$ bond formation. ${ }^{[9,10,16]}$ The liberated hydrogen bromide regenerates the catalyst precursor $\mathrm{R}_{4} \mathrm{NBr}$ from neutralization with $\mathrm{R}_{4} \mathrm{NOH}$. These individual events provide the molecular basis for selective catalysis within a defined bromine(-1/I) catalysis manifold. Over-oxidation to an unreactive bromine catalyst in an oxidation state higher than +1 could never be detected. For example, isolated $\mathbf{4}$ does not react with excess $m C P B A$, and treatment of $\mathrm{Bu}_{4} \mathrm{NBr}$ with 1-5 equivalents of $m C P B A$ does not provide bromine over-oxidation, but decomposition of the oxidant instead. ${ }^{[14]}$

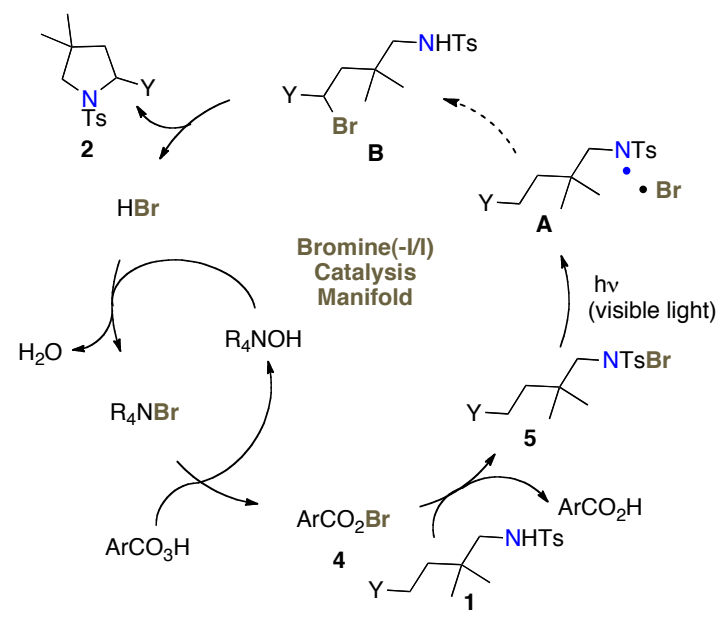

Figure 2. Mechanistic proposal: exploiting $\mathrm{Br}$ catalyst stability for a $\mathrm{Br}(-\mathrm{I} / \mathrm{l})$ catalysis manifold. $\mathrm{Y}=$ aryl, alkoxy; $\mathrm{Ar}=3-\mathrm{Cl}_{-}-\mathrm{C}_{6} \mathrm{H}_{4}$.

In contrast to the successful formation of pyrrolidines, the corresponding $1,3-$ or $1,4-\mathrm{C}-\mathrm{H}$ abstractions to aziridines or azetidines, respectively, do not constitute viable reaction pathways. For these derivatives, however, exposure to the $\mathrm{Br} / m \mathrm{~m}$ PBA system afforded an unexpected result. When exploring the reaction with 3-phenylpropanyl-1-amine $6 \mathbf{a}$ as substrate, a single product was obtained in $86 \%$ yield, which was identified as the $\mathrm{N}$-sulfonyloxaziridine 7 a (Scheme 3 ). This overall sequence thus represents a new one-pot approach to the important class of $\mathrm{N}$-sulfonyloxaziridines, which depict unique oxidants within the synthetic arsenal of modern organic chemistry. ${ }^{[17]}$ Moreover, this synthesis represents a rare case in halogen catalysis, in which oxidants are generated within a catalytic oxidative transformation. ${ }^{[18]}$ The reaction was rationalized to consist of two individual consecutive oxidation events. In the absence of available hydrogen atoms for a kinetically competent $\mathrm{C}-\mathrm{H}$ abstraction the initially formed $\mathrm{N}$ bromo species $\mathbf{5}$ undergoes an $\alpha$-elimination to the aldimine $\mathbf{C}$. The oxaziridine formation is then accomplished by oxidation of the intermediary aldimine with the terminal oxidant $m$ CPBA. ${ }^{[19]}$ Importantly, attempts to conduct the same transformation with catalytic amounts of iodine or $\mathrm{Bu}_{4} \mathrm{NI}$ did not succeed demonstrating a superior performance of bromine over iodine.

The involvement of the $\mathrm{N}-\mathrm{Br}$ intermediate $\mathbf{5}$ and the absence of the alternative benzylic bromination product is important as it confirms that the reactions from Figure 2 do proceed through a Hofmann-Löfler pathway and do not derive from the potential 
direct benzylic $\mathrm{C}-\mathrm{H}$ bromination under conventional radical conditions. The oxaziridine formation from linear sulfonamides 6 is a general reaction as displayed in Scheme 3 with 15 examples. Standard $N$-tosyl phenylpropylamide gives the corresponding oxaziridine $\mathbf{7 b}$ in $60 \%$ yield as a single oxidation product. Variation of the arene substituent provides the products 7c-d (59-70\%), while the sulfonyl variation includes mesyl (7e, 44\%), nosyl (7f, 65\%) and 2-thiophenylsulfonyl (7g, 64\%). A phenyl ether derivative $7 \mathrm{~h}$ could also be accessed conveniently $(68 \%)$.
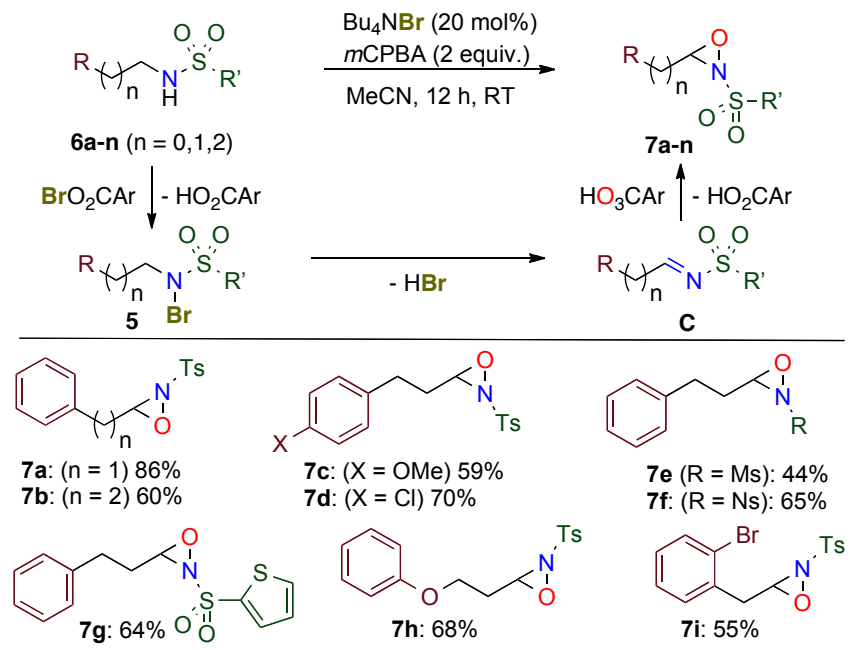

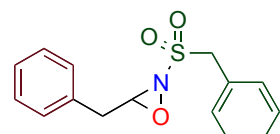

$7 \mathrm{j}: 72 \%$

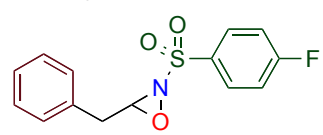

$7 \mathrm{~m}: 60 \%$

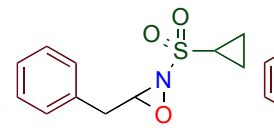

$7 \mathrm{k}: 44 \%$

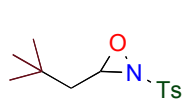

$7 n: 85 \%$

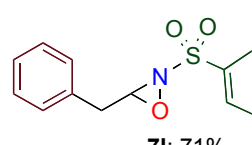

7I: $71 \%$

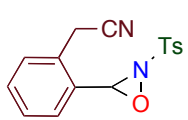

7o: $97 \%$

Scheme 3. Oxaziridine synthesis from linear N-sulfonamides: scope.

With respect to the variation of the $N$-tosyl phenylethylamide motif $\mathbf{6 a}$, its 2-brominated arene derivative $\mathbf{7} \mathbf{i}$ was accessed in $55 \%$ yield and structurally characterized by solid state X-ray analysis. ${ }^{[20]}$ Variation of the sulfonyl group includes the benzylsulfonyl derivative $(\mathbf{7 j}, \mathbf{7 2} \%)$ and the cyclopropylsulfonyl derivative $(\mathbf{7 k}, 44 \%)$. Although potentially labile under reaction conditions involving free radicals, the two transformations proceed with chemoselectivity in favor of the oxaziridine formation. The reaction scope further includes a 2naphthylsulfonyl (7I, 71\%), a 4-fluorobenzenesulfonyl (7m,60\%) and an aliphatic neopentyl substitution $(\mathbf{7 n}, 85 \%)$ in the final product. Usually, oxaziridines were never formed under any conditions for the examples of pyrrolidine formation from Scheme 1. A single exception was encountered for the nitrilesubstituted derivative $60^{[21]}$ This outcome is due to the increased acidity of the $\alpha$-hydrogens, which prevents hydrogen atom transfer and thus leads to imine and ultimately oxaziridine formation.

In summary, protocols for unprecedented bromine(-I/I)catalysis in $\mathrm{C}-\mathrm{H}$ aminative functionalization and oxaziridine

formation have been designed. The reactions proceed within defined homogeneous catalytic cycles and rely on an engineered bromine $(\mathrm{I})$ catalyst that is directly accessed from oxidation with the terminal oxidant mCPBA. For the first time in halide catalysis, the crucial $\mathrm{N}$-halogenated intermediate as the initiator of the radical $\mathrm{C}-\mathrm{H}$ functionalisation could be isolated and characterized. These accomplishments will be an instrumental guide for the development of general bromine catalysts and complimentary radical $\mathrm{C}-\mathrm{H}$ transformations.

\section{Acknowledgements}

Financial support was provided by the Spanish Ministry for Economy and Competitiveness and FEDER (CTQ2017-88496R grant to K. M., and Severo Ochoa Excellence Accreditation 2014-2018 to ICIQ, SEV-2013-0319). The authors are grateful to the CERCA Program of the Government of Catalonia and to COST Action CA15106 "C-H Activation in Organic Synthesis (CHAOS)".

\section{Keywords: Amination - Bromine • C-H Functionalization • Catalysis $\cdot$ Hofmann-Löffler Reaction}

[1] a) M. Uyanik, K. Ishihara, ChemCatChem 2012, 4, 177; b) P Finkbeiner, B. J. Nachtsheim, Synthesis 2013, 45, 979.

[2] a) J. U. Jeong, B. Tao, I. Sagasser, H. Hennigen, K. B. Sharpless, J. Am. Chem, Soc. 1998, 128, 6844; b) S. Minakata, Acc. Chem. Res. 2009, 42, 1172.

[3] P. Chavez, J. Kirsch, C. H. Hövelmann, J. Streuff, M. MartínezBelmonte, E. C. Escudero-Adán, E. Martin, K. Muñiz, Chem. Sci. 2012, 3, 2375 .

[4] a) K. Muñiz, Pure Appl. Chem. 2013, 85, 755 (2013); b) For non-related Bromine(III) chemistry: M. Ochiai, K. Miyamoto, T. Kaneaki, S. Hayashi, W. Nakanishi, Science 2011, 332, 448.

[5] a) E. Fattorusso, O. Taglialatela-Scafati (Eds.) Modern Alkaloids. WileyVCH, Weinheim, 2007; b) D. O'Hagan, Nat. Prod. Rep. 2000, 17, 435.

[6] a) T. Brückl, R. D. Baxter, Y. Ishihara, P. S. Baran, Acc. Chem. Res. 2012, 45, 826; b) B. G. Hashiguchi, S. M. Bischof, M. M. Konnick, R. A. Periana, Acc. Chem. Res. 2012, 45, 885.

[7] For C-H aminations: a) J. L. Jeffrey, R. Sarpong, Chem. Sci. 2013, 4 4092; b) R. Hili, A. K. Yudin, Nature Chem. Biol. 2006, 4, 283; c) H. M. L. R. Davies, M. S. Long, Angew. Chem. Int. Ed. 2005, 44, 3518; d) T. A. Ramirez, B. Zhao, Y. Shi, Chem. Soc. Rev. 2012, 41, 931; e) J. L. Roizen, M. E. Harvey, J. Du Bois, Acc. Chem. Res. 2012, 45, 911; f) F. Collet, C. Lescot, P. Dauban, Chem. Soc. Rev. 2011, 40, 1926.

[8] a) C. Martínez, K. Muñiz, Angew. Chem. Int. Ed. 2015, 54, 8287; b) P. Becker, T. Duhamel, C. J. Stein, M. Reiher, K. Muñiz, Angew. Chem. Int. Ed. 2017, 56, 8004.

[9] a) A. W. Hofmann, Ber. Dtsch. Chem. Ges. 1883, 16, 558; b) K. Löffler, Ber. Dtsch. Chem. Ges. 1910, 43, 2035.

[10] a) M. E. Wolff, Chem. Rev. 1963, 63, 55; b) R. S. Neale, Synthesis $1971,1$.

[11] It is interesting to compare this context in main group catalysis with the recent case of non-related $\mathrm{C}-\mathrm{H}$ functionalization using conventional transition metal $\mathrm{Rh}$ and emerging Co catalysis. For reviews on $\mathrm{Rh}$ : a) $\mathrm{P}$. B. Arockiam, C. Bruneau, P. H. Dixneuf, Chem. Rev. 2012, 112, 5879; Co: b) M. Moselage, J. Li, L. Ackermann, ACS Catal. 2016, 6, 498.

[12] M. Srebnik, Synth. Commun. 1989, 19, 197

[13] L. Candish, M. Freitag, T. Gensch, F. Glorius, Chem. Sci. 2017, 8, 3618.

[14] Please see Supporting Information for further details.

[15] A. Md. Hashem, A. Jung, M. Ries, A. Kirschning, Synlett1998, 195.

[16] L. R. Reddy, B. V. S. Reddy, E. J. Corey, Org. Lett. 2006, 8, 2819.

[17] a) F. A. Davis, B.-C. Chen, Chem. Rev. 1992, 92, 919; b) V. A. Petrov, G. Resnati, Chem. Rev. 1996, 96, 1809. 
[18] a) M. Uyanik, H. Hayashi, K. Ishihara, Science 2014, 345, 291; b) B. J. Nachtsheim, Science 2014, 345, 270.

[19] F. A. Davis, O. D. Stringer, J. Org. Chem. 1982, 47, 1774.

[20] X-ray crystallographic data for compound $7 \mathbf{i}$ has been deposited with the Cambridge Crystallographic Data Centre database (http://www.ccdc.cam.ac.uk/) under code CCDC 1583024.
[21] For mechanistically non-related oxidative $\alpha$-oxidation of carbonyl compounds under iodine catalysis, see ref. 18a and (a) M. Uyanik, $\mathrm{H}$. Okamoto, T. Yasui, K. Ishihara, Science 2010, 328, 1376 (b) M. Uyanik D. Suzuki, T. Yasui, K. Ishihara, Angew. Chem. 2011, 123, 5443; Angew. Chem. Int. Ed. 2011, 50, 5331. 
Entry for the Table of Contents (Please choose one layout)

Layout 1:

\section{COMMUNICATION}

Br enters catalysis! New

homogeneous bromine catalysis by combining an ammonium bromide with $m C P B A$ as oxidant enables the first catalytic variant of the classic bromine-mediated Hofmann-Löffler reaction. The same conditions transform sulfonamides into oxaziridine derivatives within a single operation.

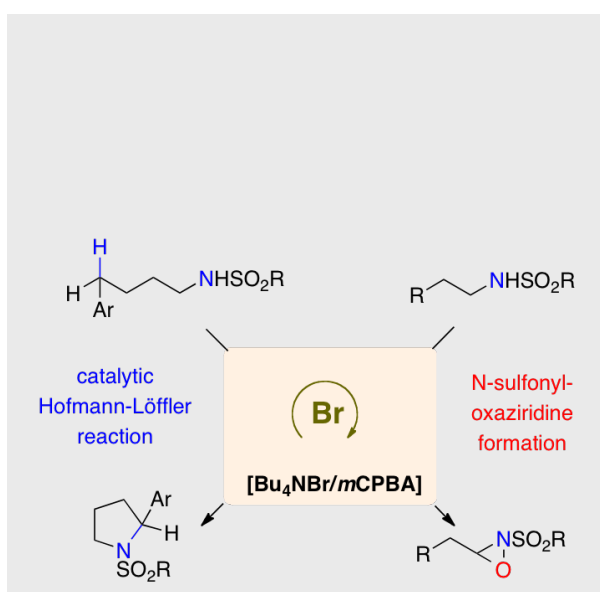

P. Becker, T. Duhamel, C. Martínez, K Muñiz*

Page No. - Page No.

Designing Homogeneous Bromine Redox Catalysis for Selective Aliphatic C-H Bond Functionalization 